\title{
Nematopsis sp. (Apicomplexa: Eugregarinida) em Mytella guyanensis (Lamarck, 1819) (Bivalvia: Mytilidae) da Região Estuarina do Rio Cachoeira, Ilhéus, Bahia, Brasil
}

Thiago Ramos PINTO' Guisla BOEHS²

\section{Correspondência para:}

Guisla Boehs - Universidade Estadual de Santa Cruz, Departamento de Ciências Biológicas, Rodovia Ilhéus-ltabuna, km 16, 45.650-000, Ilhéus, Bahia; gboehs@uesc.br; Fone: (73) 3680-5307

Recebido para publicação: 05/09/2006 Aprovado para publicação: 13/12/2007

\author{
1 - Departamento de Ciências Agrárias e Ambientais da Universidade Estadual de \\ Santa Cruz, Ilhéus - BA \\ 2 - Departamento de Ciências Biológicas da Universidade Estadual de Santa Cruz, \\ Ilhéus - BA
}

\begin{abstract}
Resumo
Exemplares do molusco Mytella guyanensis (Bivalvia: Mytilidae) da região estuarina do Rio Cachoeira (Ilhéus, Bahia) foram investigados quanto à freqüência de ocorrência e sítios de infecção por protozoários do gênero Nematopsis Schneider, 1892 (Apicomplexa: Eugregarinida: Porosporidae), no período entre agosto de 2005 e julho de 2006 . Em 480 animais analisados, medindo entre 34,5 e $74,9 \mathrm{~mm}$ de altura (Média: 55,4 mm; DP \pm 6,7), 387 apresentaram em seus tecidos o parasito e nestes, os locais com maior freqüência de ocorrência do Nematopsis foram as brânquias (298 animais) e o manto (248). O parasito foi também observado na glândula digestiva e na musculatura. Alguns indivíduos gravemente infectados apresentaram modificações na conformação das brânquias e do manto.
\end{abstract}

\section{Introdução}

A Bahia apresenta uma região costeira com aproximadamente $1100 \mathrm{~km} \mathrm{de}$ extensão, com estuários localizados ao longo de 40 bacias hidrográficas. ${ }^{1}$ Entre estas, encontra-se a Bacia Hidrográfica do Rio Cachoeira (BHRC), que conflui com os rios Itacanoeira (ou Fundão) e Santana, formando um estuário num local conhecido como Coroa Grande, no Município de Ilhéus. ${ }^{2}$ Moluscos de interesse econômico (e.g., ostreídeos, mitilídeos, venerídeos) habitam o infralitoral raso, os baixios entremarés e os manguezais da região, e várias espécies são consumidas e comercializadas pelas comunidades ribeirinhas locais.

Mytella guyanensis (Lamarck, 1819), com distribuição do México ao Peru, no Oceano Pacífico, e da Venezuela ao Brasil, no Atlântico ${ }^{3}$, habita as regiões entremarés de manguezais, onde utiliza o bisso para fixar-se nas raízes subterrâneas. No Brasil, esse molusco é conhecido, entre outros nomes vulgares, como "sururu" e "bico de ouro". Similarmente ao que ocorre com outros mitilídeos, $M$. guyanensis tem potencial para o cultivo, atividade que está sendo experimentalmente iniciada em alguns locais do litoral brasileiro. Em nível local, apesar de extraída nos bancos naturais para o consumo humano, o cultivo dessa espécie ainda não foi iniciado. Também, pouco se conhece sobre a biologia e os parasitos associados com esse molusco na região.

O phylum Apicomplexa inclui várias espécies de protozoários conhecidos por parasitarem moluscos marinhos de importância comercial, principalmente bivalves. ${ }^{4}$ Esse grupo inclui a ordem Eugregarinida (Léger, 1900), família Porosporidae Labbé, 1899, com os gêneros Porospora Schneider, 1875 e Nematopsis Schneider, $1892^{5}$. Esses parasitos, também conhecidos como gregarinas, utilizam o trato intestinal de crustáceos marinhos no estágio de esquizogonia e moluscos bivalves nas fases de gametogonia e esporogonia ${ }^{2}$. Nos 
moluscos, parasitam tecidos de diferentes órgãos como o manto, as gônadas, as brânquias e a glândula digestiva. ${ }^{6,7,8} \mathrm{Os}$ oocistos (esporos das gregarinas contendo em seu interior um ou mais esporozoítos uninucleados e vermiformes) ocorrem dentro de um fagócito do molusco, e podem deslocar-se no tecido conjuntivo para vários órgãos, sendo, porém, mais freqüentes nas brânquias. ${ }^{4}$

Os registros de Nematopsis em bivalves incluem hospedeiros como: Crassostrea rhizophorae ${ }^{9,10,11}$, C. gigas $^{10}, C$. virginica $^{8}, M$. guyanensis ${ }^{5}$, Perna perna ${ }^{12}, P$. canaliculus $^{13}$, Cerastoderme edule ${ }^{6,7}$ e Ruditapes decussatus ${ }^{6}$. No Brasil, Nematopsis foi registrado na ostra-do-mangue C. rhizophorae da Baía de Todos os Santos (BA) ${ }^{9}$, Ilha de Santa Catarina $(\mathrm{SC})^{10}$ e estuário do Rio Jaguaribe $(\mathrm{CE})^{11}$, na ostra-do-Pacífico C. gigas $^{10}$ da Ilha de Santa Catarina, no mexilhão P. perna ${ }^{12}$ da Lagoa de Itaipu (RJ) e no sururu $M$. guyanensis ${ }^{5}$ da região estuarina do Rio Amazonas, a que Azevedo e Matos denominaram de N. mytella. A forma, a dimensão, a espessura da parede e a morfologia do opérculo dos oocistos de Nematopsis são, segundo esses últimos autores, as mais evidentes diferenças morfológicas encontradas entre as várias espécies deste gênero.

Até o momento, não existem estudos sobre o potencial patogênico dessas gregarinas em humanos. No presente estudo, avaliou-se a freqüência de ocorrência e a localização deste protozoário no sururu $M$. guyanensis da região estuarina da Bacia do Rio Cachoeira (Ilhéus, BA).

\section{Material e Método}

Os exemplares de $M$. guyanensis foram coletados manualmente em áreas de manguezal na região estuarina da Bacia do Rio Cachoeira, na confluência dos rios Santana, Cachoeira e Fundão (14\%48'23"S; $\left.39^{\circ} 02^{\prime} 47^{\prime \prime} \mathrm{W}\right)$ e a montante, no Rio Cachoeira propriamente dito $\left(14^{\circ} 49^{\prime} 04^{\prime \prime} \mathrm{S}\right.$; $\left.39^{\circ} 03^{\prime} 44^{\prime \prime} \mathrm{W}\right)$, entre agosto de 2005 e julho de 2006, totalizando 480 animais coletados em 24 amostragens quinzenais (20 animais/ coleta). Um monitoramento paralelo na região, no mesmo período, evidenciou temperatura da água variando entre $24 \mathrm{e}$ $31^{\circ} \mathrm{C}$ e salinidade, a depender do estágio da maré, com valores entre zero e 23.

Os animais foram transportados em recipientes etiquetados contendo água do mar até o seu processamento nos laboratórios de Oceanografia Biológica e Histologia Animal da Universidade Estadual de Santa Cruz (Ilhéus). Após feita a medição da altura (eixo dorso-ventral), utilizando-se um paquímetro manual com precisão de 0,01 $\mathrm{mm}$, procedeu-se a evisceração de cada exemplar. Realizada a análise macroscópica, retirou-se uma secção dos tecidos, em sentido diagonal na massa visceral de cada espécimen, amostrando-se o manto (e gônadas - impregnadas no manto), brânquias, tubo digestório, glândula digestiva e musculatura. O material biológico foi fixado em solução de Davidson ${ }^{14} \mathrm{e}$ transferido, decorridas aproximadamente 30 horas, para etanol $70 \%$. O processamento histológico seguiu rotina de desidratação em série alcoólica crescente, diafanização em xilol, impregnação em parafina, obtenção de cortes com cerca de 7 micrômetros de espessura por microtomia, coloração por Hematoxilina de Harris e Eosina (HE), e montagem sob lamínula. As lâminas foram examinadas em microscopia de luz e fotomicrografadas.

\section{Resultados e Discussão}

Os exemplares de Mytella guyanensis mediram entre 34,5 e $74,9 \mathrm{~mm}$ de altura (Média: 55,4 mm; DP \pm 6,7; $\mathrm{n}=480$ ). Não houve qualquer evidência macroscópica indicando a presença do parasito. A freqüência de ocorrência de Nematopsis sp. foi de 80,6\% (387 animais/480) e variou de $40 \%$ (outubro/2005 e março/2006) a 100\% (novembro/2005 e abril/2006) (Figura 1). As brânquias foram o local com a maior freqüência de ocorrência de infecção (298 animais $/ 480=62,1 \%$ ), seguidas pelo manto $(248 / 480=51,7 \%)$, glândula digestiva (47/ 


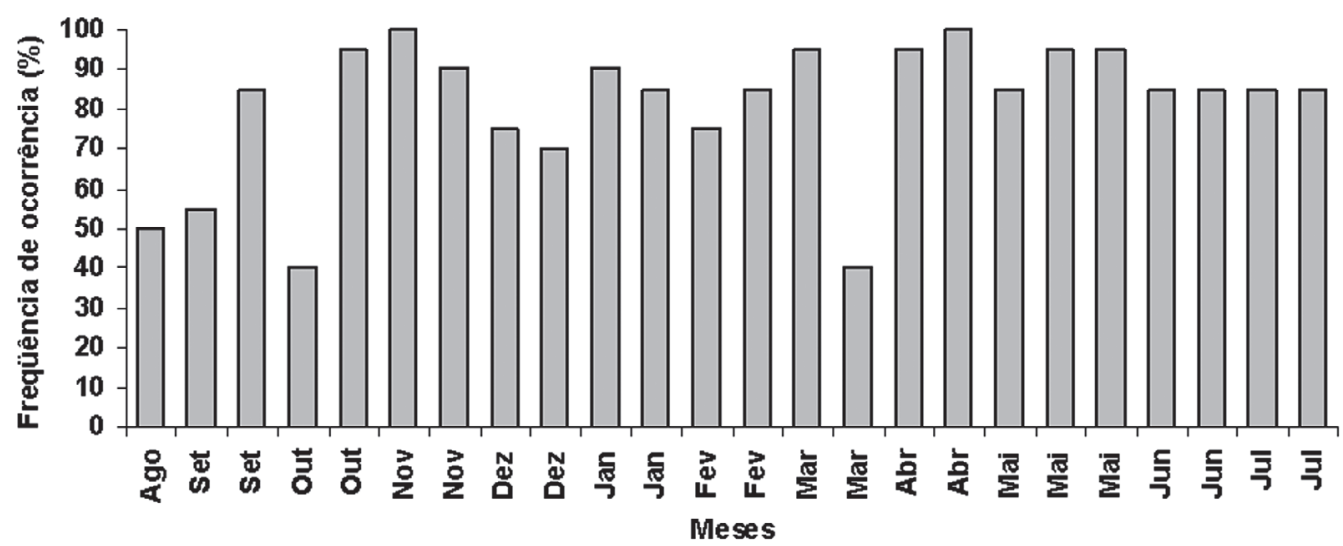

Figura 1 - Freqüência de ocorrência de Nematopsis sp. em Mytella guyanensis da região estuarina da Bacia do Rio Cachoeira, Ilhéus (BA), no período de agosto 2005 a julho de $2006(n=480)$

$480=9,8 \%)$ e músculo $(32 / 480=6,6 \%)$. Em 47,3\% dos animais parasitados, ocorreu infecção simultânea de dois ou mais locais.

$\mathrm{O}$ número de oocistos por fagócito variou de um a aproximadamente 20 , entretanto, a situação mais comum foi de 1 3 oocistos/fagócito (Figura 2). O morfotipo observado trata-se possivelmente de $N$. mytella, espécie observada no mesmo hospedeiro na região estuarina do Rio Amazonas por Azevedo e Matos ${ }^{5}$, que verificaram a ocorrência de três a 19 oocistos / fagócito. Um estudo ultraestrutural provavelmente poderá confirmar esta hipótese.

Na região sul de Portugal, Azevedo e Cachola $^{6}$ encontraram freqüência de ocorrência de $8 \%$ de Nematopsis sp. em Ruditapes decussatus e de mais de $82 \%$ em Cerastoderme edule. O parasito foi observado irregularmente distribuído no tecido conjuntivo das brânquias em ambas as espécies, tratando-se, segundo os autores, de um mesmo morfotipo. Possivelmente tratando-se da mesma espécie observada por esses autores, Carballal et al. ${ }^{7}$ registraram freqüências entre 30 e 100\% em C. edule da região da Galícia (Espanha). Além da ocorrência nas brânquias, o parasito foi observado também no manto, no músculo e na glândula digestiva. Na Nova Zelândia, Jones $^{13}$ registrou ocorrência de cerca de $80 \%$ de Nematopsis nas brânquias e palpos do bivalve Perna canaliculus. Winstead et al. ${ }^{8}$ verificaram freqüências de 24 a $90 \%$ de $N$. ostrearum e $N$. prytherchi no tecido conjuntivo próximo ao estômago, no manto e nas brânquias de ostras Crassostrea virginica da Flórida (EUA). No Brasil, Nascimento et al. ${ }^{9}$ observaram, na ostra-do-mangue $C$. rhizophorae da Baía de Todos os Santos (BA), freqüência de até $100 \%$, com ocorrência do parasito principalmente no músculo adutor. Em ostras desta mesma espécie do estuário do Rio Jaguaribe (CE), foram observadas freqüências entre 60 e $100 \%$, com ocorrência preferencial nas brânquias e no manto ${ }^{11}$. $\mathrm{Na}$ Ilha de Santa Catarina (SC) Sabry e Magalhães ${ }^{10}$ registraram freqüências respectivas de $60 \%$ e $70 \%$ em ostras cultivadas C. rhizophorae e C. gigas, sendo que os sítios de ocorrência foram o manto, lúmen do tubo digestório, brânquias e glândula digestiva. Lima, Abreu e Mesquita ${ }^{12}$ registraram freqüência de ocorrência de 36\% nas brânquias do mexilhão Perna perna na Lagoa de Itaipu (RJ). Em M. guyanensis da região estuarina do Rio Amazonas, foi verificada freqüência de ocorrência de 21\% e presença de N. mytella em vários locais, principalmente nas brânquias. ${ }^{5}$ Observa-se que os resultados do presente estudo são concordantes com os anteriormente realizados, tanto em relação às ocorrências geralmente altas, quanto aos principais sítios de infecção nos moluscos. Com poucas exceções $^{5,8,13}$, na maior parte destes trabalhos não foi feita a determinação da espécie de Nematopsis.

Quanto aos danos que estas gregarinas 

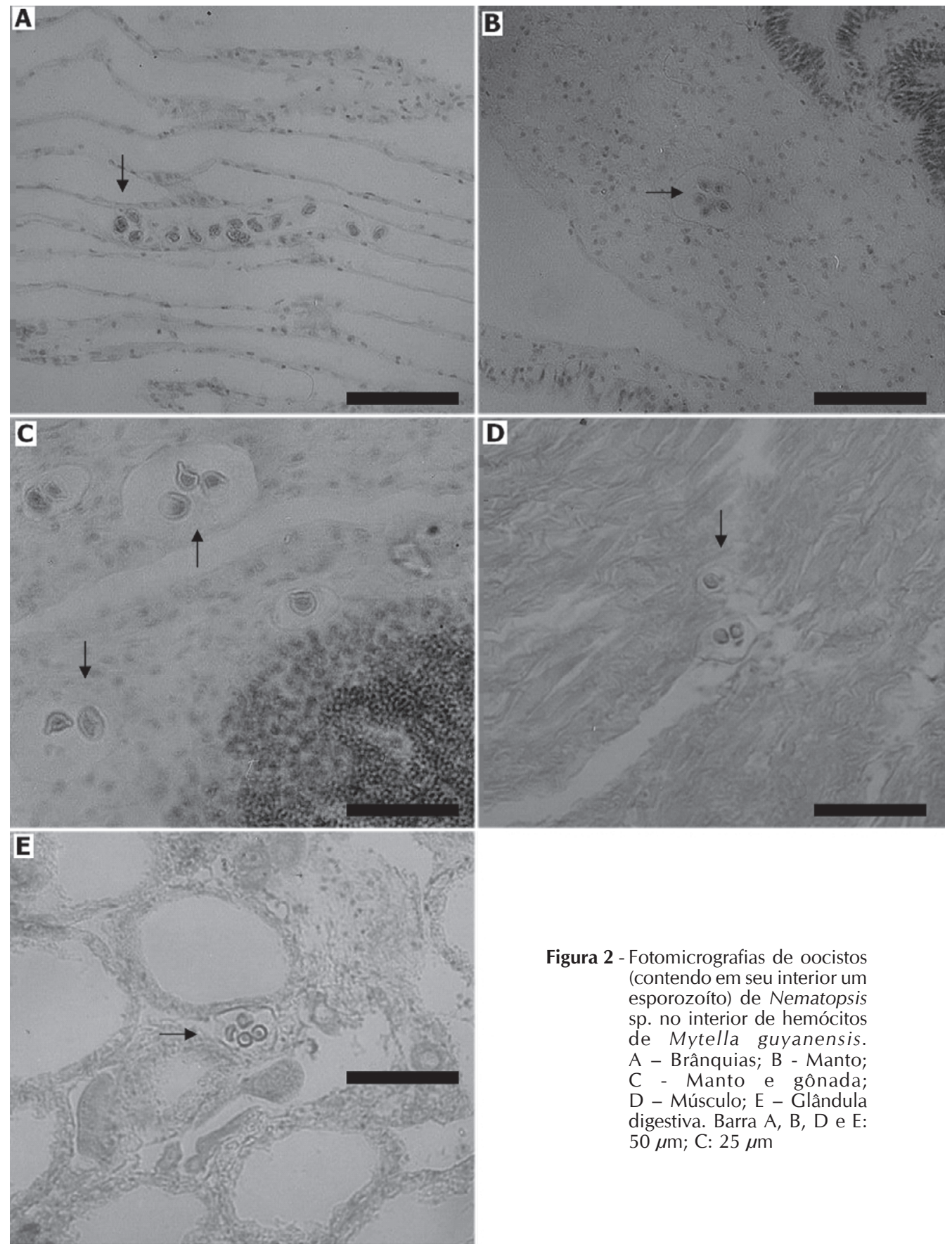

Figura 2 - Fotomicrografias de oocistos (contendo em seu interior um esporozoíto) de Nematopsis sp. no interior de hemócitos de Mytella guyanensis. A - Brânquias; B - Manto; C - Manto e gônada; D - Músculo; E - Glândula digestiva. Barra A, B, D e E: $50 \mu \mathrm{m} ; \mathrm{C}: 25 \mu \mathrm{m}$

podem causar nos moluscos, geralmente os hospedeiros apresentam apenas reação hemocitária focal., 4,7,10,11 Entretanto, estudos ultraestruturais em $M$. guyanensis parasitada por N. mytella evidenciaram lise celular com completa degradação do fagócito infectado ${ }^{5}$ o que foi também observado no bivalve $C$. edule, no qual verificou-se, também, destruição de tecidos das brânquias e mortalidade devido a esta infecção. ${ }^{6}$ No presente estudo, foram observadas alterações na morfologia das brânquias e do manto em alguns animais severamente infectados, porém, 
representando um pequeno número de casos. Esse fato sugere que este parasito não está causando, até o momento, danos significativos aos estoques naturais do sururu M. guyanensis na região estuarina do Rio Cachoeira. Porém, a alta freqüência de ocorrência registrada deve ser levada em conta na implantação de cultivos desta espécie, em cujos sistemas o Nematopsis poderá representar riscos, principalmente se ocorrer concomitantemente com outros parasitos/patógenos.

\title{
Nematopsis sp. (Apicomplexa: Eugregarinida) in Mytella guyanensis (Lamarck, 1819) (Bivalvia: Mytilidae) from estuarine region of the Cachoeira river, Ilhéus, Bahia, Brazil
}

\begin{abstract}
Individuals of the clam Mytella guyanensis (Bivalvia: Mytilidae) from the estuarine region of the Cachoeira river (Ilhéus, Bahia) have been analyzed to detect the frequency of occurrence and infected sites by protozoans of the genus Nematopsis Schneider, 1892 (Apicomplexa: Eugregarinida: Porosporidae), in the period between August 2005 and May 2006. A total of 480 individuals were analyzed, measuring between 34.5 and $74.9 \mathrm{~mm}$ of height (Average: $55.4 \mathrm{~mm}$; DP \pm 6.7 ). Nematopsis were found in 387 of them, occurring more frequently within the gills (298 animals) and the mantle (248). The parasite was also observed in the digestive gland and the musculature. Some severely infected individuals presented modifications in the conformation of the gills and the mantle.
\end{abstract}

\section{Referências}

1- RAMOS, S. Exploração de Moapen Tagelus plebeius (Lightfoot, 1786) (Mollusca: Pelecypoda) no Estuário do Rio Cachoeira: estratégia de sobrevivência humana e valorização da seção estuarina de Ilhéus, Bahia. 2002. 125 f. Dissertação (Mestrado em Desenvolvimento Regional e Meio Ambiente) - Universidade Estadual de Santa Cruz, Ilhéus, 2002.

2- SCHIAVETTI, A.; SCHILLING, A. C.; OLIVEIRA, H. T. Caracterização Sócio-ambiental da Bacia Hidrográfica do Rio Cachoeira Sul da Bahia, Brasil. In: SCHIAVETTI, A.; CAMARGO, A. F. M. Conceitos de bacias hidrográficas: teorias e aplicações. Ilhéus: Editus, 2002. p. 141-161.

3- RIOS, E. C. Seashells of Brazil. Rio Grande: Editora da Fundação Universidade do Rio Grande, 1994. $492 \mathrm{p}$.

4- BOWER, S. M.; McLADDERY, S. E.; PRICE, M. I. Synopsis of Infectious Diseases and Parasites of Commercially Exploited Shellfish. Annual Review of Fish Diseases, v. 4, p. 1-199, 1994.

5- AZEVEDO, C.; MATOS, E. Description of Nematopsis mytella sp. (Apicomplexa), Parasite of the Mussel Mytella guyanensis (Mytelidae) from the Amazon Estuary and Description of its Oocysts. European Journal of Protistology, v. 35, p. 427-433, 1999.
6- AZEVEDO, C.; CACHOLA, R. Fine structure of the apicomplexa oocyst of Nematopsis sp. of two marine bivalve molluscs. Diseases of Aquatic Organisms, v. 14, p. 69-73, 1992.

7- CARBAlLAL, M. J. et al. Parasites and Pathologic Conditions of the Cockle Cerastoderma edule Populations of the Coast of Galicia (NW Spain). Journal of Invertebrate Pathology, v. 78, p. 87-97, 2001.

8- WINSTEAD, J. T.; VOLETY, A. K.; TOLLEY, S. G. Parasitic and symbiotic fauna in oyster (Crassostrea virginica) collected from the Caloosahatchee River and Estuary in Florida. Journal of Shellfish Research, v. 23, p. 831-840. 2004.

9- NASCIMENTO, I. A. et al. Pathological Findings in Crassostrea rhizophorae from Todos os Santos Bay, Bahia, Brazil. Journal of Invertebrate Pathology, v. 47, p. 340-349, 1986.

10- SABRY, R. C.; MAGALHÃES, A. R. M. Parasitas em ostras de cultivo (Crassostrea rhizophorae e Crassostrea gigas) da Ponta do Sambaqui, Florianópolis, SC. Arquivo Brasileiro de Medicina Veterinária e Zootecnia, v. 57, p. 194-203, 2005. Suplemento 2.

11- SABRY, R. C.; GESTEIRA, T. C. V.; BOEHS, G. First record of parasitism in the mangrove oyster Crassostrea rhizophorae (Bivalvia: Ostreidae) at Jaguaribe River estuary - Ceará, Brazil. Brazilian Journal of Biology, v. 67, n. 4, p. 631-637, 2007. 
12- LIMA, F. C.; ABREU, M. G.; MESQUITA, E. F. M. Monitoramento histopatológico de mexilhão Perna perna da lagoa de Itaipu, Niterói, RJ. Arquivo Brasileiro de Medicina Veterinária e Zootecnia, v. 53, n. 2, p. 203-206, 2001.

13- JONES, J. B. Nematopsis n.sp. (Sporozoa: Gregarinia) in Perna canaliculus. N. Z. Journal of Marine and Freshwater Research, v. 9, n. 4, p. 567568, 1975.

14- SHAW, B. L.; BATTLE, H. I. The gross and microscopic anatomy of the digestive tract of the oyster Crassostrea virginica (Gmelin), Canadian Journal of Zoology, v. 35, p. 325-347, 1957. 